\title{
Multi-strand coronal loop model and filter-ratio analysis
}

\author{
Sofiane Bourouaine and Eckart Marsch \\ Max-Planck-Institut für Sonnensystemforschung, 37191 Katlenburg-Lindau, Germany \\ bourouaine@mps.mpg.de
}

\begin{abstract}
We model a coronal loop as a bundle of seven separate strands or filaments. Each of the loop strands used in this model can independently be heated (near their left footpoints) by Alfvén/ion-cyclotron waves via wave-particle interactions. The Alfvén waves are assumed to penetrate the strands from their footpoints, at which we consider different wave energy inputs. As a result, the loop strands can have different heating profiles, and the differential heating can lead to a varying cross-field temperature in the total coronal loop. The simulation of TRACE observations by means of this loop model implies two uniform temperatures along the loop length, one inferred from the 171:195 filter ratio and the other from the 171:284 ratio. The reproduced flat temperature profiles are consistent with those inferred from the observed EUV coronal loops. According to our model, the flat temperature profile is a consequence of the coronal loop consisting of filaments, which have different temperatures but almost similar emission measures in the cross-field direction. Furthermore, when we assume certain errors in the simulated loop emissions (e.g., due to photometric uncertainties in the TRACE filters) and use the triple-filter analysis, our simulated loop conditions become consistent with those of an isothermal plasma. This implies that the use of TRACE/EIT triple filters for observation of a warm coronal loop may not help in determining whether the cross-field isothermal assumption is satisfied or not.
\end{abstract}

Subject headings: Sun: solar corona, waves, Sun: magnetic fields, particle acceleration

\section{Introduction}

In our recent papers Bourouaine et al. (2008a, b) (hereafter referred to as BR2008a; BR2008b) we modelled a coronal loop as one single flux tube (or monolithic loop) in which the confined plasma is heated by ion-cyclotron/Alfvén waves via the resonant wave absorption 
mechanism. When considering Coulomb collisions in that model, it turned out that they are not strong enough to maintain local thermal equilibrium, and it was found that the electron temperature profile is different from the ion profile. Furthermore, it was shown that the warm coronal loop (e.g., $T \leq 1.5 \mathrm{MK}$ ), which has a quasi-uniform electron temperature profile and enhanced density (relative to the static model by Serio et al. 1981), is the consequence of a uniform cross-section of the flux tube forming the loop. Modelling of this type of loop profile with a uniform temperature was motivated by the many observations of EUV (Extreme Ultraviolet) loop emissions (using the high spatial resolution imaging provided by TRACE (Lenz et al. 1999; Aschwanden et al. 1999, 2000).

Under the assumption that the loops have a single cross-field temperature, techniques based on filter-ratio analysis have been employed to infer the electron temperature along the EUV coronal loops (Noglik and Walsh 2007; Noglik et al. 2008; Aschwanden et al. 2008; Schmelz et al. 2009). Most of the obtained results indicate that the warm loops have roughly constant temperatures along much of their lengths. Furthermore, it was found that their densities are roughly uniform and much larger than those predicted by static models with uniform heating (Aschwanden et al. 2001; Winebarger et al. 2003). However, it was concluded that static loop models with footpoint heating may produce flat temperature profiles and enhanced apex densities, but in the case of long loops the theoretical enhancement in density was found to be not large enough as compared to the observed values.

It is believed that the difficulty of reproducing the observed properties of TRACE loops with static models lies in that they are not in thermal equilibrium. Therefore, it was suggested that impulsive heating caused by nanoflares could explain the observed loop characteristics. However, in such process, numerical simulations showed that the single-loop cooling would appear simultaneously in both the TRACE $171 \AA$ and $195 \AA$ filters for a few seconds (Reeves and Warren 2002). This cooling time is much shorter than the lifetime of the active-region loops observed by TRACE, which generally live for several hours while maintaining their high densities and flat temperature profiles.

Therefore, it has been suggested that the relevance of the impulsive heating heavily relies on the assumption of a multi-strand or multi-thread nature of coronal loops, as it is illustrated in Fig. 1, and these strands are at different stages of heating and cooling exhibiting a broad differential emission measure (DEM) distribution.

The concept of multi-stranded loops is supported by many observations which exploit the high spatial resolution of TRACE and indicate that an observed "fat" single loop could be composed of many small-scale filaments having most probably different temperatures across the magnetic field (Schmelz et al. 2001, 2003, 2005; Martens et al. 2002). Hydrodynamic models based on multi-stranded loops have been suggested and used to calculate 
High resolution

Low resolution

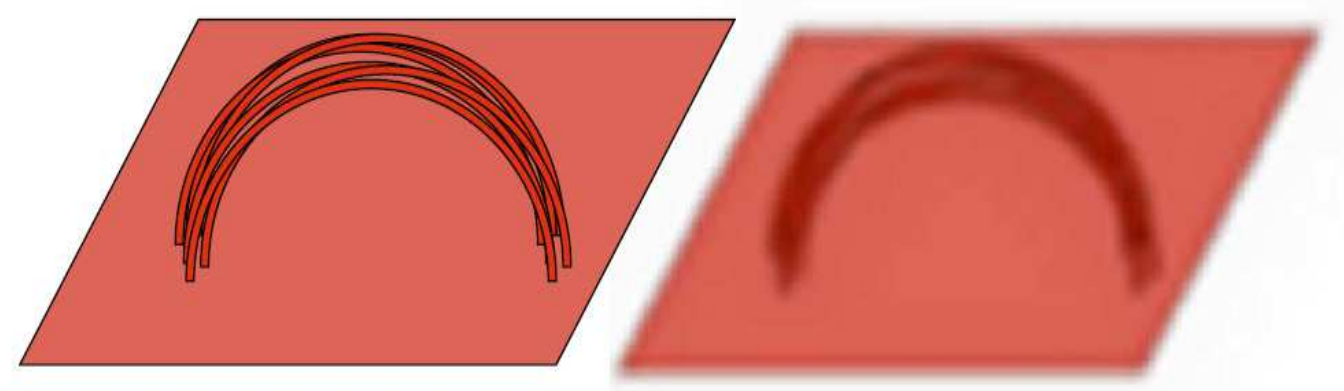

Fig. 1.- Coronal model loop that consists of a bundle of many magnetic strands

numerically the flare emission (Warren 2006; Reeves et al 2007). Other multi-strand coronal loop models involving nanoflare heating have been proposed to simulate EUV coronal loops (e.g., Cargill and Klimchuk 1997; Warren et al. 2002; Sarkar and Walsh 2009).

The lack of observations of very fine-scale structures at scales below the TRACE resolution still permits the possibility that the observed loops may be composed of very thin "strands or filaments" which have different temperature and density profiles. As a result, the observed loop intensity could be caused by an ensemble of emissions from many unresolved strands.

Reale and Peres (2000) produced the observed features of warm coronal loops by assuming them to consist of a bundle of static, uniformly heated strands. Their model, however, implements dense and hot strand $(\sim 5 \mathrm{MK})$ to reproduce the flat TRACE filter ratios. This seems to contradict previous observations which indicate that, generally, hot loops are not co-spatial with relatively cool loops (Sheeley 1980; Habbal et al. 1985; Brooks et al. 2007).

Following the concept of a multi-strand structure of coronal loops, we will also model the observed TRACE loop as a bundle of seven isolated strands. However, concerning loop heating we propose here that the strands are heated through the dissipation of parallel propagating ion-cyclotron/Alfvén waves via resonant wave absorption. The same kinetic model that has been used in the papers BR2008a and BR2008b to describe the heating of a monolithic loop is now applied to the heating of an individual small-scale strand. Thus various fine structures can have different temperatures and density profiles. Therefore, an observation of a neighbouring set of filaments with a low-resolution instrument could lead to an apparently fat loop, which yet is simply composed of a finite number of strands. Since in the corona the cross-field heat transport is very weak, and as the plasma is dominated by 
the magnetic pressure, we can assume that separate loop strands are thermally isolated.

Once we have determined the different plasma profiles of single loop strands, we can synthesize the total emission of the composite model loop, thereby assuming its observation as a monolithic loop in the TRACE/EIT filters, and thus we can extract its temperature by adopting the filter-ratio technique. As a result we find that, if the coronal loop is composed of unresolved filaments having different temperatures and roughly identical emission measures across the coronal loop, a quasi-uniform temperature is inferred along the loop length.

The paper is organized as follows: in Sec. 2 we model a TRACE/EIT coronal loop as a bundle of seven separate filaments heated through the dissipation of high-frequency Alfvén waves. We assume different wave energy inputs at the footpoints of the different strand. Then in Sec. 3, we synthesize the emission of the modelled coronal loop in the three TRACE passbands, and thus we can derive the loop temperature from two filter ratios 171:195 and 171:284. Finally, we discuss the obtained results and conclude in Sec. 4.

\section{Multi-stranded coronal loop model}

It is widely believed that the small-scale reconnection events occurring in the lower corona (or chromosphere) can be a source of high-frequency plasma waves, namely waves having frequencies comparable to the local ion-gyrofrequency (Axford and McKenzie 1992; Axford et al. 1999). But only recently, by using STEREO, Helios and Cluster data, have ioncyclotron waves clearly been detected at different distances in the solar wind (see Jian et al. 2008). Previously, theoretical fluid models (Marsch and Tu 1997; Tu and Marsch 1997; Hackenberg et al. 2000; He et al. 2008) have shown that the propagation of such waves into the corona and their subsequent absorption can lead to efficient ion heating in open magnetic structures (such as coronal funnels). Thus kinetic models could explain the rapid temperature increase in the transition region and corona (Vocks 2002; Vocks and Marsch 2002). Therefore, this wave heating mechanism may also be relevant to the closed structures like coronal loops. Indeed, that formation of hot coronal loops can be a consequence of the dissipation of ion-cyclotron waves along the closed magnetic fields confining the plasma in the loops, was shown by Bourouaine et al. $(2008 \mathrm{a}, \mathrm{b})$ using kinetic models.

Furthermore, intuitively we may think that the random spatial occurrence of reconnection events may generate ion-cyclotron waves with different amounts of energy at various locations. The dissipation of the waves along neighbouring closed field lines originating there can lead to plasma heating and acceleration on those field lines, thus producing small-scale plasma filaments or magnetic loop strands (which may in the extreme case have scales com- 
parable to the ion-skin depth but certainly below the the present TRACE spatial resolution). These filaments may have differential temperature and density profiles, and the observations of neighbouring sets of such filaments with low-resolution instruments may reveal an apparent fat coronal loop that really is composed of a finite number of those small-scale strands (see Fig. 1).

In this paper, we will represent a coronal loop as an ensemble of seven separated loop strands or filaments. Each strand is modelled as an electron-proton plasma confined within a semi-circular cylindric and symmetric flux tube. All the strands (numbered by index $i$ ) are assumed to have the same length $(L=63 \mathrm{Mm})$ and the same local width, $w_{i}(s)$. We suggest that these strands are close to each other and situated in planes that are perpendicular to the solar surface. The flux tubes of these strands expand similarly with height, i.e., the filaments have the same expansion factor, $\Gamma=1.48$. This means that each strand has a varying cross-section which expands from the strand footpoints (situated at the transition region) to the strand top and satisfies formula (13) in the paper BR2008a.

The loop strands are heated by dissipation of non-dispersive Alfvén/ion-cyclotron waves which are assumed to be injected at the left footpoints of the strands and propagate along the field lines. A power-law spectrum is assumed for these waves at the left footpoint, $s=0$, such that

$$
\mathfrak{B}_{\omega}^{i}(s=0)=\alpha_{i}\left[\omega \ln \left(\Gamma^{2}\right)\right]^{-1} \operatorname{erg} \mathrm{cm}^{-3} \mathrm{~s}^{-1}, \quad \mathrm{i}=1,2 . .7
$$

where $\mathfrak{B}_{\omega}^{i}$ denotes the wave spectral energy density. The parameter $\alpha_{i}$ which corresponds to the footpoint wave intensity of each loop strand is listed in Tab. 1]

The heating associated with the dissipation of the Alfvén waves via resonant waveparticle interactions is a fully kinetic process and does not involve collisions (which are rare in solar corona) for the dissipation of the waves. In such process, the ions have to obey the cyclotron resonance condition, $\omega-k_{\|} v_{\|}-\Omega_{i}=0$, with the parallel wave vector $k_{\|}$, wave frequency $\omega$, and ion gyrofrequency $\Omega_{i}(s)=q_{i} B(s) / m_{i}$, which varies along the strand with the spatial coordinate $s$ through the magnetic field strength $B(s)$. Here $q_{i}$ is the charge of ion species $i$ and $m_{i}$ its mass.

Since the cross section of the magnetic flux tube (chosen for each strands) expands by more than twice its footpoint value up to the middle of the loop strand, the waves can be strongly damped at distances not far from the left footpoint, and thus heat the protons at different positions near the left footpoint (leading to quasi-footpoint heating). More details on this dissipation mechanism are given in previous papers (Vocks and Marsch (2002), BR2008a).

All the loop strands have the same boundary conditions, i.e., their electron densities at 
Table 1: The parameter $\alpha_{i}$ corresponds to the wave power density of strand " $i$ ", defined by its maximum proton temperature shown in Fig. 1.

\begin{tabular}{|c|c|c|}
\hline Strand & $T_{\text {Max }}(\mathrm{MK})$ & $\alpha_{i} \times 10^{5}$ \\
\hline 1 & 0.7 & 0.5 \\
2 & 0.84 & 0.7 \\
3 & 1.2 & 1.1 \\
4 & 1.4 & 1.4 \\
5 & 1.66 & 1.9 \\
6 & 1.95 & 2.5 \\
7 & 2.1 & 3.0 \\
\hline
\end{tabular}

the footpoints $(s=0, s=L)$ are given by $N_{e}(s=0)=N_{e}(s=L)=5 \times 10^{9} \mathrm{~cm}^{-3}$, and their electron temperatures by $T(s=0)=T(s=L)=2 \times 10^{5} \mathrm{~K}$.

For the sake of simplification we considered the lower transition region (or the upper chromosphere) to be the boundary for our modelled loop. This is justified since at these altitudes the plasma can be assumed to be still fully ionized, and therefore we avoid the complexity of considering the neutral part of the solar atmosphere. Also, the heating mechanism acting on the base of the corona might be different from the one proposed here for coronal loops. Recently, it was for example suggested that dissipation of low-frequency Alfvén waves could help in overcoming the low initial temperature in the lower transition region (Bourouaine et al. 2008c). Furthermore, the universal upflows driven by spicules (or chromospheric jets) can play a significant role in supplying the lower corona with hot plasma (De Pontieu et al. 2009).

At $t=0$, we consider as initial condition that all strands are in hydrostatic equilibrium with a similar initial state, i.e., the strands are cool with isothermal temperature, $T=0.2 \mathrm{MK}$ and have relatively small apex densities. After about ten thousand seconds all the strands reach their steady final states, and they get filled with hot plasma due to the evaporation process and plasma acceleration, and thus form an unresolved TRACE or SOHO/EIT loop. It was shown in BR2008a that the wave energy flux density needed to heat a loop strand to

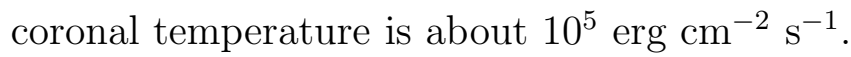

The temperature and density profiles of each loop strand are plotted in Fig. 2. As expected, the higher the wave energy input the more energy is transferred to the plasma loop strand. The Alfvén waves heat the protons via ion-cyclotron-wave absorption, and thus due to proton-electron collisions also the electrons can be heated. The proton temperatures increase up to their maximum values close to a distance of $\approx 20 \mathrm{Mm}$ where the dissipation of the waves ceases. The proton temperatures tend to decrease under the effect of heat 
conduction and electron-proton energy exchange by collisions. However, the electron temperature is roughly equal to the proton temperature as long as the latter is smaller than one mega kelvin. But when the proton temperature exceeds this value, the electron temperature remains at smaller values due to strong electron heat conduction. Therefore, the electron and proton temperatures in the strands "1, 2" roughly overlap. While, in the case of strands "3, 4, 5, 6, 7", the protons are hotter than the electrons, and electrons show a quasi-uniform heating.

In this kinetic model, the loop strands can be filled with plasma via two mechanisms, wave-induced particle acceleration and chromospheric plasma evaporation (caused by heat conduction from the corona). However, wave-particle acceleration is the dominant process to fill the loop-like structures with protons, since the proton heat conduction is small, and the electron inertia is neglected in our approximation. Thus, the plasma dynamics of the loop is only related to the proton inertia. As a result, we see that the densities in big parts of the loop strands do not differ by much. Consequently, the strands have a roughly similar emission measure $\left(E M=N^{2} w\right)$ across the loop.

Therefore, the modelled coronal loop (represented by a multi-strand loop) has a varying cross-field electron temperature, which ranges from 0.6 to $1.6 \mathrm{MK}$ at its top, but only a small cross-field density variation (or slight cross-field emission-measure variation). The next section is devoted to the analysis of the emission of the modelled coronal loop, as it would be observed in the three EUV channels at 171, 195 and $284 \AA$ available in the TRACE imager or SOHO/EIT.

\section{Filter-ratio analysis}

In the following section we introduce the term "isothermal temperature", $T_{\text {iso }}$, which simply means the observationally inferred loop temperature, when assuming a single crossfield temperature and using the filter-ratio technique.

It is possible to synthesize the total emission of the modelled coronal loop from the summed strand emissions. Each strand intensity can easily be computed from its density and temperature profile by using the following emission measure relation:

$$
I_{i}(s)=w_{i}(s) N_{i}^{2}(s) G^{\lambda}\left(T_{i}(s)\right), \quad \mathrm{i}=1,2 . .7
$$

where $I_{i}(s)$ is the intensity of the strand "i" given per unit optical length (units of $\mathrm{DN} \mathrm{s}^{-1}$ pixel $^{-1}$ ) at position $s$ along the strand. The symbols $T_{i}$ and $N_{i}$ denote, respectively, the strand electron temperature (in units of kelvin) and density (in units $\mathrm{cm}^{-3}$ ), and $G^{\lambda}(T$ ) is 
the response function for each filter centered around wavelength $\lambda$; it is plotted in frame (a) and the filter ratio in panel (b) of Fig. 3 .

The strand emissions, $I_{i} / w_{i}$, for the three filters are plotted in Fig. 4. It seems that the brightest strands in the $171 \AA$ emission are those labelled with numbers 3,4 , and 2 (ordered from the highest to lowest emission). The $171 \AA$ filter is more sensitive to the strands having temperatures from 0.7 MK to 1.2 MK. Therefore, the first three strands mainly contribute to the total emission of the coronal loop in the $171 \AA$ passband (see Fig. $4 \mathrm{~d}$ ). Moreover, from Fig. 4b it seems that the $195 \AA$ filter is sensitive to all strands, except for those labelled with numbers 1 and 2. This is because this filter is sensitive to the plasma temperatures from $1 \mathrm{MK}$ to $1.4 \mathrm{MK}$. However, less visibility of the loop strands is expected in the filter $284 \AA$ (see Fig. 4c), since this filter is sensitive only to hot plasma with $T \geq 1.6 \mathrm{MK}$, and thus, the total loop also appear with a small emission in this filter (see Fig. 4d). Furthermore, in the realistic case when the background has to be accounted for, this modelled loop can hardly be seen in the $284 \AA$ passband, since its emission would be contaminated strongly by background effects. Therefore, we can classify our modelled multi-strand loop as a warm EUV loop $(T \leq 1.5 \mathrm{MK})$ in terms of visibility in the TRACE or SOHO/EIT filters.

Fig. 5f displays the emission ratios of the modelled coronal loop versus its loop length. Interestingly, the emission ratio 171:195 (indicated by a solid line) varies slightly between two values, 1 and 3 inside the loop. However, the emission ratio 171:284 (indicated by dot lines) spans between the values between 10 and 12. As we will show, the variations in these two emission ratios are still not large enough to produce variations in the isothermal temperatures along the loop length.

Based on the obtained emission ratios, and by making use of the ratios between the response functions plotted in Fig. 3b, we can extract the isothermal temperatures that would be inferred from TRACE or EIT observations of our modelled coronal loop. The inferred loop temperature profiles from the two filter ratios, 171:195 and 171:284, are plotted in Fig 6a. Notice that these inferred temperatures differ from the computed averaged temperature profile.

It turns out that there are two distinct temperature profiles that can be inferred from the filter-ratio analysis for a simulated TRACE or EIT coronal loop. This is a consequence of the multi-thermal property of our modelled coronal loop. It seems as if the use of two different filter ratios in the TRACE or EIT coronal loop observations allows us to determine whether these observed loops are isothermal or multi-thermal. If the two filter ratios do not provide the same temperature profile, this may be an indication that the cross-field single temperature assumption was not satisfied. This conclusion may explain the results obtained by Schmelz et al. (2003). The authors analysed 10 coronal loops that were clearly visible 
in the 171,195 and $284 \AA$ passbands of the EIT. They showed that in each case of the used background substraction method two different uniform temperatures were obtained, one from the 171:195 ratio and the second for the 195:284 ratio. The authors suggested that the single cross-field temperature assumption could provide a misleading loop temperature.

However, we argue here that, in case of warm loops $(T \leq 1.5 \mathrm{MK})$, it is difficult to infer the temperature from the filter ratio 195:284. This is because the ratio function 195:284 in Fig. 3b peaks at a temperature $T \sim 1.26 \mathrm{MK}$, and for any ratio value that spans between $\sim 1$ and $\sim 26$, there are two corresponding temperatures; one is above $1.26 \mathrm{MK}$ and the other below it (non-unique solution). Therefore, in the case of warm coronal loops, it is difficult to constrain the temperature value to be chosen. For that reason, we did not use here the filter ratio 195:284 to determine the loop temperature.

By assuming a line-of-sight depth of $10^{10} \mathrm{~cm}$, as considered by Lenz et al. (1999), we estimate the emission measure from the filter ratio (171:195) and (171:284) (see Fig 6b). The emission measure profile implies an enhanced density inside the loop, and the estimated EM for the first filter ratio is comparable to the one obtained by Lenz et al. (1999), however, a higher EM could be obtained if we considered the filter ratio (171:284).

For warm coronal loops, the use of the triple filters 171/195/284 $\AA$ may not help to decide if these loops are isothermal across the field or not. This is because these loops may not appear clearly in the filter $(284 \AA$ ) (Chae et al. 2002). Their emission can be strongly affected by the background emission, and therefore, if their appearance in the filter (284 $\AA$ ) leads to a temperature uncertainty of the order of ten percent, then it would be difficult to discriminate between the two temperature profiles obtained from the two filter ratios (171:195) and (171:284).

Fig. 7 displays EM-loci plots at some position along the modelled loop. It is clear that the curves do not exactly intersect in a single point. However, these curves are approaching each other in a narrow temperature interval, and in some papers (Schmelz et al. 2009; Tripathi et al. 2009) the authors suggested that this narrow crossing area in the EM curves is due the not strictly isothermal nature of the loop along the line of sight. In the general case, this also can be a misleading interpretation. Although the modelled loop is completely multi-thermal, the curves only intersect in a narrow temperature interval around $T \sim 1.2 \mathrm{MK}$.

For example, by considering the photometric uncertainties determined by Patsourakos and Klimchuk (2007), our simulated coronal loop is found to be consistent with an isothermal plasma. The authors define a new quantity based on triple-filter analysis called, the average logarithmic difference, which is 


$$
\Delta \log [E M(T)]=\frac{1}{3}\left\{\log \frac{E M_{171}}{E M_{195}}+\log \frac{E M_{171}}{E M_{284}}+\log \frac{E M_{195}}{E M_{284}}\right\} .
$$

This quantity considers all three of the possible channel combinations. It vanishes when the emission measures, obtained from the three possible filter-ratios, are identical at the isothermal temperature, $T_{i s o}$. This is the case for accurate observations of an isothermal plasma. However, in real measurements the errors have to be account for, which are characterized by an uncertainty $\Delta \log (E M)_{\text {uncertain }}$.

The uncertainty in each emission-ratio is obtained from the determination of the uncertainty in the corresponding intensity-ratio. The intensity measurements are affected by systematic errors due to imperfect knowledge of the photometric calibration, and therefore, the uncertainties associated with relative photometry errors among the 171, 195, and $284 \AA$ channels have to be characterized. Patsourakos and Klimchuk (2007) have estimated the av-

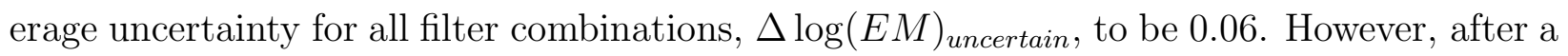
CDS/TRACE/EIT inter-calibration study in a quiet-Sun region, Brooks and Warren (2006) found that the $284 \AA$ filter sensitivity could be underestimated by a factor of approximately 3 , and therefore, the uncertainty $\Delta \log (E M)_{\text {uncertain }}$ could reach the value 0.63 .

The isothermal interpretation may only be valid when $\Delta \log (E M)$ is smaller than $\Delta \log (E M)_{\text {uncertain. }}$ In Fig. 8 we plot the $\Delta \log [E M(T)]$ curve at some locations along the loop. There are absolute minima near $1.2-1.4 \mathrm{MK}$ which are below the error bar. As we can see, although our simulated loop is multi-thermal, the loop can be considered as isothermal according to the argument given above. This means that with the triple-filter method suggested by Patsourakos and Klimchuk (2007), it is not possible to confirm the isothermal interpretation assumed for the observed loops.

There is an interesting behaviour in the inferred temperature profiles of our modelled coronal loop, which is that these profiles are quasi-uniform along the loop length (see Fig.6a). This notion seems to fit the TRACE coronal loop features as discussed in the introduction.

According to our modelled loop, the flatness in the temperature profile is consequence of loop consisting of filaments, which have similar emission measures, $E M_{i}$ and different temperatures in the cross-field direction. In such case, the overall loop intensity ratios, at a given loop position, simply would reduce to the ratios of the response functions summed over the number of the loop strands, $N_{s}$, within this coronal loop position, i.e., to the ratios

$$
R=\frac{\sum_{i=1}^{N_{s}} G^{\lambda_{1}}\left(T_{i}\right)}{\sum_{i=1}^{N_{s}} G^{\lambda_{2}}\left(T_{i}\right)}
$$

where, $\lambda_{1}$ and $\lambda_{2}$ are the wavelengths of considered filters. 
Therefore, flat temperature profiles, which are often obtained when using the filterratio technique, can even occur in the case when the loop consists of a small number of strands (forming a real discrete loop structure) with features described above. However, Weber et al. (2005) suggested that the flatness of the temperature profile may also occur if a loop has continuum cross-field structure of varying cross-field temperature (i.e., constant broad differential emission measure (DEM)). In such a case, the emission ratio method is biased toward the ratio of the integrated response functions over a broad temperature interval. This is nothing but a particular example of equation (4), when assuming a large number of strands (forming a continuum structure).

\section{Conclusion}

In this work we synthesized the emission of seven strands which together are assumed to constitute a coronal loop as seen in a low-resolution TRACE or SOHO/EIT observation. Each of the loop strands used in the model is heated by Alfvén/ion-cyclotron waves via waveparticle interactions. This process leads to proton heating in the electron-proton plasma, and due to electron-proton collisions the electrons can then be heated as well up to a certain temperature which is below or equal to the proton temperature. It turns out that the plasma, in case of hot loop strands (where the proton temperature exceeds $>1 \mathrm{MK}$ ) is not in local thermal equilibrium and the electrons are cooler than the protons.

In our model, the Alfvén waves, which are assumed to penetrate the strands from their footpoints, are there generated with different intensities. Consequently, different heating profiles occur within each strand due to the wave absorption or heating process. Therefore, this differential heating leads to a varying cross-field temperature in the total coronal loop. Moreover, due to the ion acceleration process caused by wave-particle interactions, the model produces roughly similar electron density values (or emission measure since the strands have the same widths) across the loop.

The simulated TRACE observation of the modelled loop implies two different quasiuniform temperature profiles along the loop length, one derived from the filter-ratio 171:195 and the other for the 171:284. This flatness behaviour in the temperature profiles is consistent with results based on the filter ratio analysis applied to TRACE/EIT coronal loop. According to our model results, such flat temperature profiles can occur when the loop consists of a small number of threads (forming a discrete loop like-structure) which have different temperature profiles but rough similar emission measure, EM, across the total loop.

It is worth noting that, due to the week binary collisions between the plasma species, 
the loop plasma can be far from local thermal equilibrium if the ion temperature exceeds one mega Kelvin. Therefore, it is ideally suited for applying the kinetic description of a plasma rather than using the single fluid approach to model the coronal loop heating.

Furthermore, most of the previous hydrodynamic models invoked arbitrary heating functions in the energy equation to model the loop heating. However, our model adopts a physical

mechanism for loop heating. The considered heating process relies on wave-particle interactions described by the quasi-linear theory of the kinetic Vlasov equation. This process does not only lead to plasma heating (or ion heating) but also accelerates the ions in the loop strands due to the wave pressure, and thus, can fill these strands with hot plasma. This loop-filling mechanism, which is different from the evaporation process, is naturally involved in our model, and it turns to be important in order to reproduce the observed features of coronal EUV loops.

\section{REFERENCES}

Aschwanden, M. J., et al. 1999, ApJ, 515, 842

Aschwanden, M. J., et al. 2000, ApJ, 531, 1129

Aschwanden, M. J., et al. 2001, ApJ, 550, 1036

Aschwanden, M. J., et al. 2007, ApJ, 656, 577

Aschwanden, M. J., et al. 2008, ApJ, 680, 1477

Axford, W. I., et al. 1999, Space Sci. Rev., 87, 25

Axford, W. I. \& McKenzie, J. F., 1995, in Solar Wind Seven, Cospar Conference Series Vol.3, Pergamon Press, Oxford, pp. 1-5

Bourouaine, S., Vocks, C. \& Marsch, E. 2008, ApJ, 676, 1346

Bourouaine, S., Vocks, C. \& Marsch, E. 2008, ApJ, 680, L77

Bourouaine, S., Marsch, E. \& Vocks, C. 2008, ApJ, 684, L119

Brooks, D. H., \& Warren, H. P. 2006, ApJS, 164, 202

Brooks, D. H., et al. 2007, PASJ, 59, 691

Cargill, P. J., \& Klimchuk, J. A. 1997, ApJ, 478, 799 
Chae, J., et al. 2002, ApJ, 567, L159

De Pontieu, B., et al. 2009, ApJ, 70, L1

Habbal, S. R., Ronan, R., \& Withbroe, G. L. 1985, Solar Phys., 98, 323

Hackenberg, A., Marsch, E. \& Mann, G. 2000, Astron. \& Astrophys., 360, 1139

He, J.-S., Tu, C.-Y., \& Marsch, E. 2008, Solar Phys., 250, 147

Jian, L. K., et al. 2008, AGU meeting, A1574+

Lenz, D. D., DeLuca, E. E., Golub, L., Rosner, R., \& Bookbinder, J. A. 1999, ApJ, 517, L155

Marsch, E., \& Tu, C.-Y. 1997, Solar Phys., 176, 87

Martens, P. C. H., Cirtain, J. W., \& Schmelz, J. T. 2002, ApJ, 577, L115

Noglik, J. B., \& Walsh, R. W. 2007, ApJ, 655, 1127

Noglik, J. B., et al. 2008, ApJ, 674, 1191

Patsourakos, S., \& Klimchuk, J. A. 2007, ApJ, 667, 591

Reale, F., \& Peres, G. 2000, ApJ, 528, L45

Reeves, K. K., \& Warren, H. P. 2002, ApJ, 578, 590

Reeves, K. K., et al. 2007, ApJ, 668, 1210

Rosner, R., Tucker, W.H \& Vaiana, G.S. 1978, ApJ, 220, 643

Sarkar, A. \& Walsh, R. W. 2009, ApJ, 699, 1480

Schmelz, J. T., et al. 2009, ApJ, 691, 503

Schmelz, J. T., Nasraoui, K., Richardson, V. L., Hubbard, P. J., Nevels, C. R., \& Beene, J. E. 2005a, ApJ, 627, L81

Schmelz, J. T., Beene, J. E., Nasraoui, K., Blevins, H. T., Martens, P. C. H., \& Cirtain, J. W. 2003, ApJ, 599, 604

Schmelz, J. T. 2002, ApJ, 578, L161 
Schmelz, J. T., Scopes, R. T., Cirtain, J. W., inter, H. D., \& Allen, J. D. 2001, ApJ, 556, 896

Serio, S., Peres, G., Vaiana, G. S., Golub, L., \& Rosner, R. 1981, ApJ, 243, 288

Sheeley, N. R. 1980, Solar Phys., 66, 79

Tripathi, D., et al. 2009, ApJ, 694, 1256

Tu, C.-Y., \& Marsch, E. 1997, Solar Phys., 171, 363

Vocks, C. 2002, ApJ, 568, 1017

Vocks, C., \& Marsch, E. 2002, ApJ, 568, 1030

Warren, H. P., Amy R. Winebarger, A. R., \& Paul S. Hamilton, P. S. 2002, ApJ, 579, L41

Warren, H. P. 2006, ApJ, 637, 522

Weber, M. A., et al., 2005, ApJ, 635, L101

Winebarger, A et al. 2003, ApJ, 587, 439 

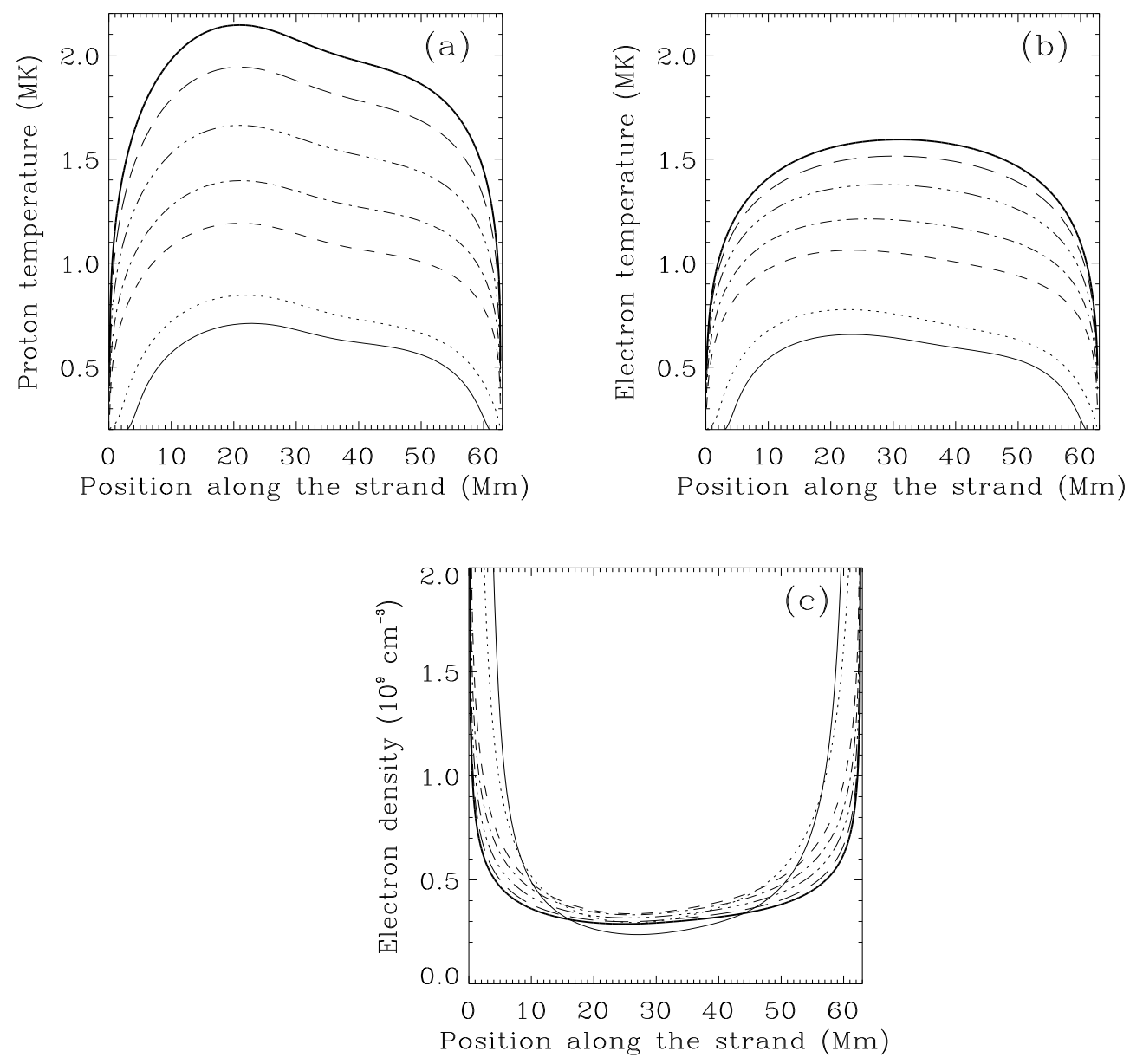

Fig. 2.- Plasma profiles for each loop strand, (a): Proton temperature, (b) electron temperature and (c) electron density. All parameters are plotted as functions of strand position. The line style (solid line) refers to strand "1", (dotted line) strand "2", (dashed line) strand "3", (dash-dotted line) strand "4", (dash-triple dotted line) strand "5", (long dashed line) strand "6" and (thick solid line) strand "7". 

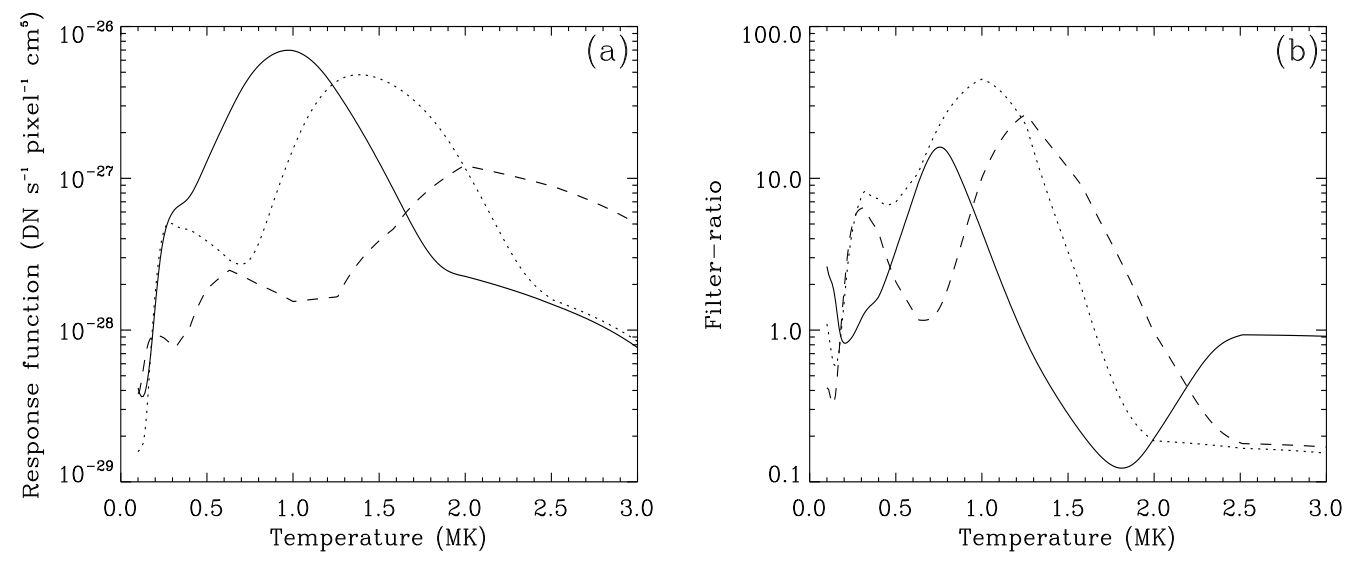

Fig. 3.- (a): The response function for each TRACE filter; filter with $171 \AA$ passband (solid line), filter with $195 \AA$ passband (dotted line) and filter with $284 \AA$ passband (dashed line). (b): The ratio between two filters; (171:195) (solid line), (171:284) (dotted line) and (195:284) (dashed line). 

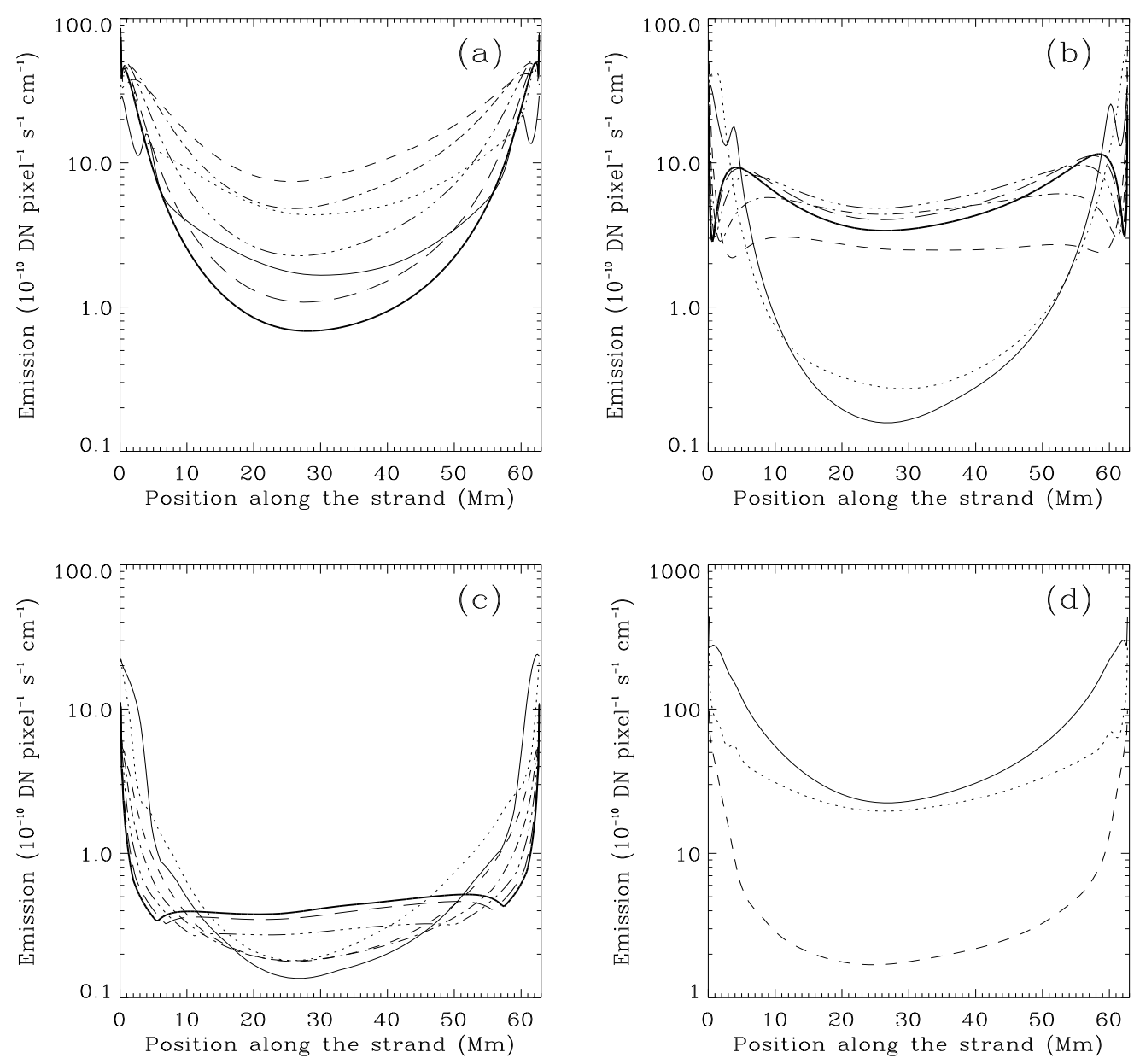

Fig. 4.- (a): Emission of each strand through the filter (171 $\AA$ ). (b): Emission of each strand through the filter $(195 \AA)$. (c): Emission of each strand through the filter $(284 \AA)$. All parameters are plotted as a function of the strand position. Each strand is represented by lines of the same style as used in Fig. 2, (d): Total loop emission through the filters $(171 \AA)$

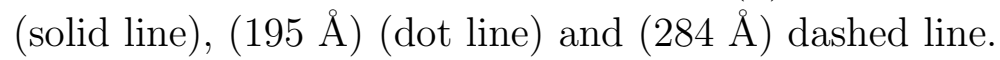



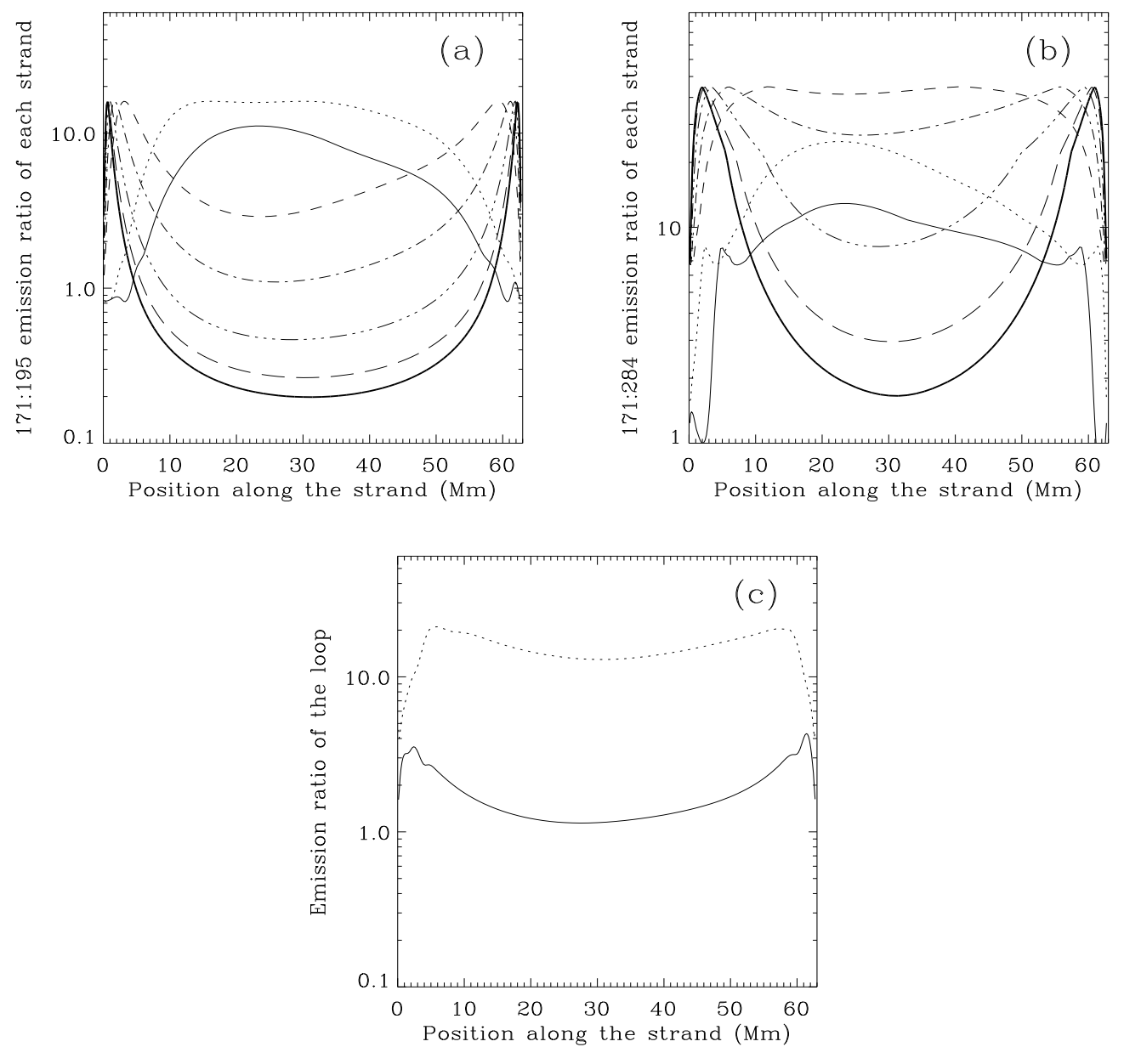

Fig. 5.- Emission ratios 171:195 (a), 171:284 (b), plotted for each strand represented by the same type of line as given in Fig. 2. (c): Averaged emission ratios of the loop, (171:195) (solid line) and (171:284) (dot line). 

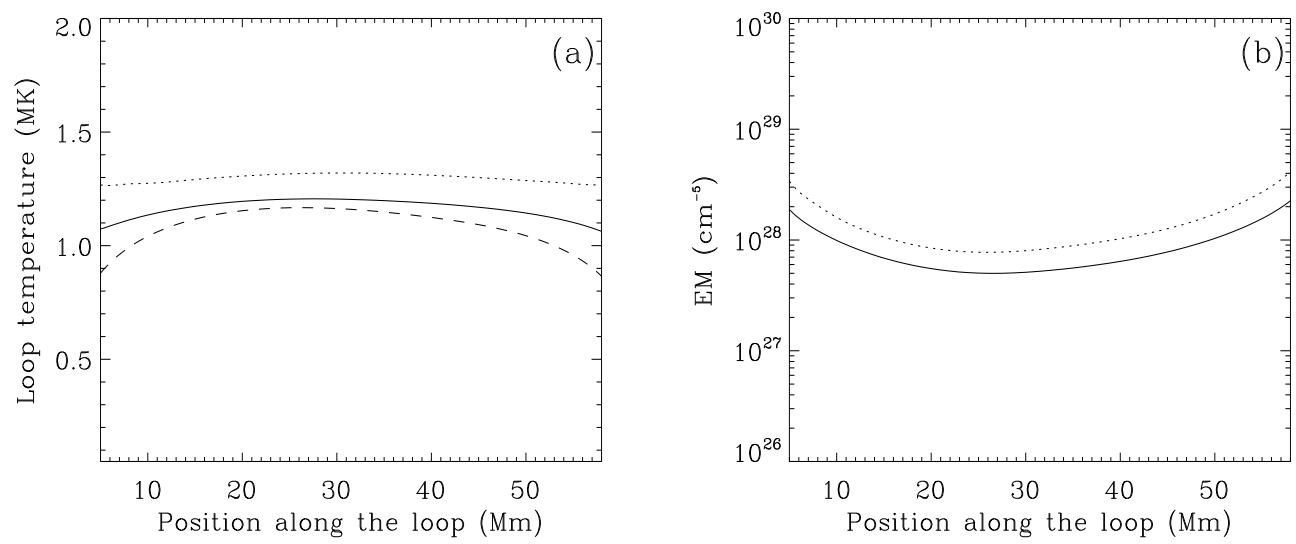

Fig. 6.- (a) and (b): Loop temperatures and loop emissions. (a): Isothermal temperature, $T_{i s o 1}$ that corresponds to the filter ratio (171:195) (solid line), $T_{i s o 2}$ that corresponds to the filter ratio (171:284) (dotted line) and the average temperature (dashed line). (b): Emission measures correspond to filter ratios (171:195) (solid line) and (171:284) (dotted line). The line of sight depth assumed here is $10^{10} \mathrm{~cm}$, a value suggested by Lenz et al. (1999) to compute the emission measure. 

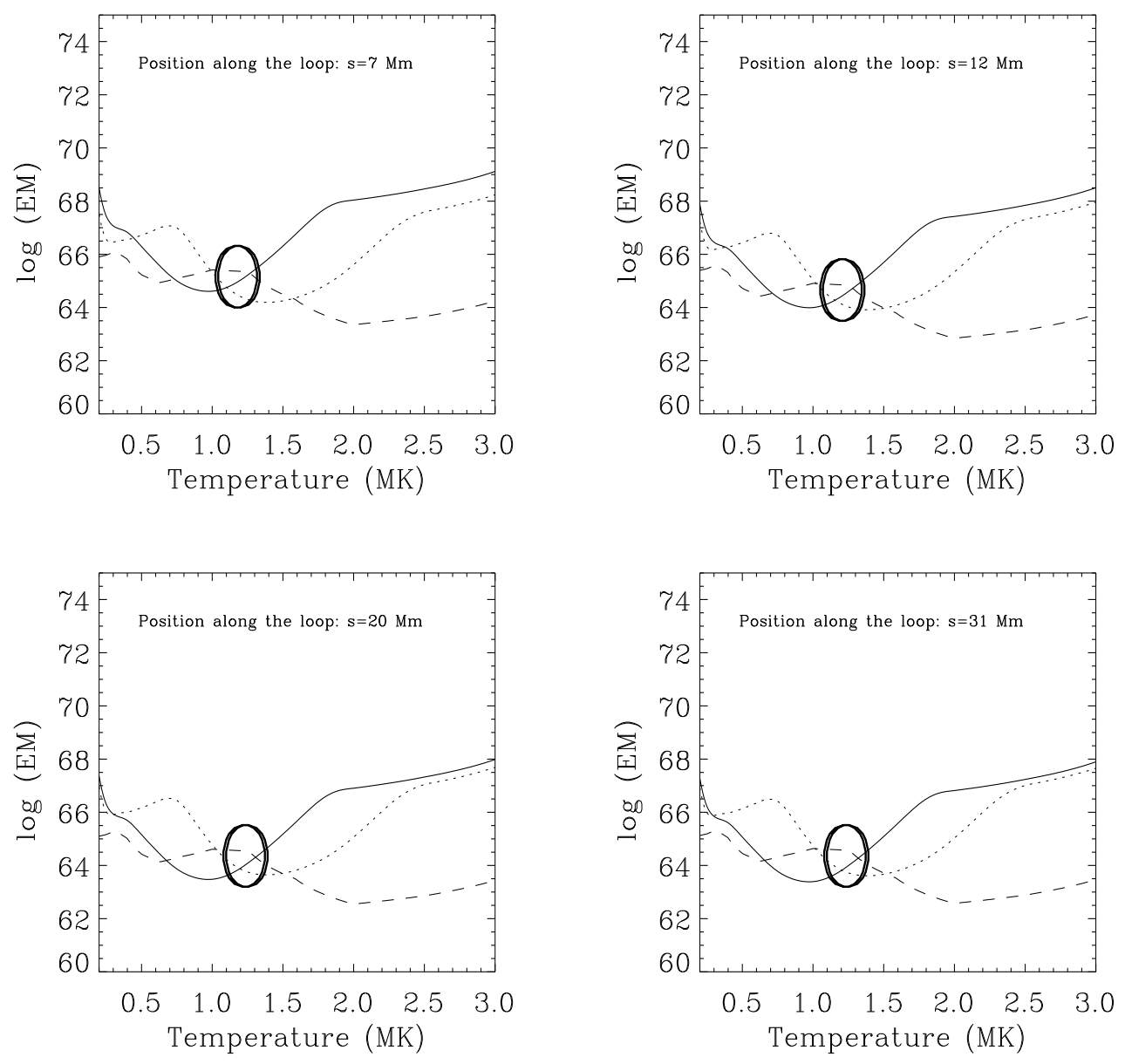

Fig. 7.- Loop emission measure curves for (171 $\AA$ ) (solid line), filter (195 $\AA$ ) (dotted line) and filter $\left(284 \AA\right.$ ) (dashed line). The line of sight depth assumed here is $10^{10} \mathrm{~cm}$, as was suggested by Lenz et al. (1999). 

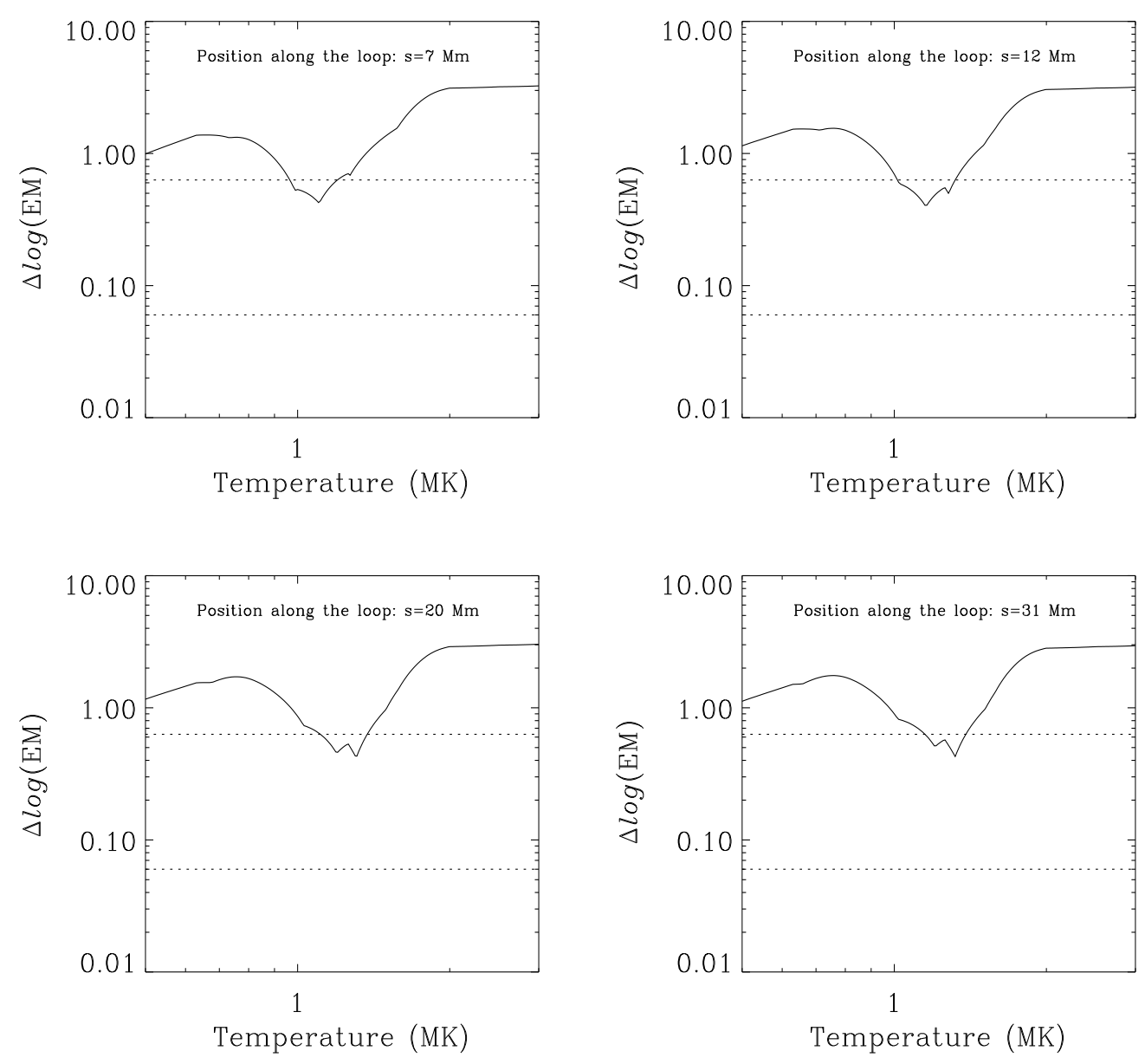

Fig. 8.- Average logarithmic difference of the isothermal emission measures versus temperature. The horizontal dashed lines correspond to the lower, respectively the upper, limit of the uncertainty of the average logarithmic difference as determined by Patsourakos and Klimchuk (2007). 